\title{
MULTI-MODELING DISSIPATIVE POTENTIAL FOR NONLINEAR VISCOELASTICITY: ANNULUS FIBROSUS APPLICATION
}

\author{
Arne Vogel, Dominique Pioletti \\ Laboratory of Biomechanical Orthopedics, EPFL, Switzerland
}

\section{Introduction}

Computational joint biomechanics requires detailed constitutive modelling for biological materials and accurate description of the strain-rate response under fast loading. In this work we present a novel dissipation potential that details the short-time component of non-linear viscoelastic response within the constitutive framework set forth by [Pioletti, 2000] for both short and long-time memory effects in finite deformation. We used descriptive material parameters whose introduction greatly simplifies the task of describing and contrasting different strain-rate sensitive materials. The particular dissipation potential proposed automatically meet the second law of thermodynamics. The procedure is applied to the annulus fibrosus under uni-axial compression.

\section{Methods}

Constitutive modelling can be separated into different time scales: an equilibrium, a short-time and a long-time contribution. The equilibrium component is modelled using classical hyperelasticity (strain-energy density), the shorttime component is describes by a dissipation potential that is explicitly dependent on strain-rate, the long-time component is not considered in this abstract. This constitutive framework enables the extension of any existing hyperelastic models to strain-rate dependent modelling (viscohyperelasticity). The proposed dissipation potential is build on the assumption that the material behaves asymptotically when subjected to relative high strain-rates and that the viscous response is isotropic. An identification algorithm is proposed to efficiently return a set of material properties. Fortunately the finite element implementation of this Kelvin-Voigt type model is straightforward.

\section{Results}

The proposed dissipation potential is capable of catching various nonlinear viscous behaviour with just four material parameters. The annulus fibrosus under uni-axial compression is appreciably well described by the proposed framework. Although, the results should not be projected in situ as our mechanical testing do not meet physiological loading conditions, one should however notice that the stress, for a given strain, increases significantly with strain-rate and that this relation is non-linear.

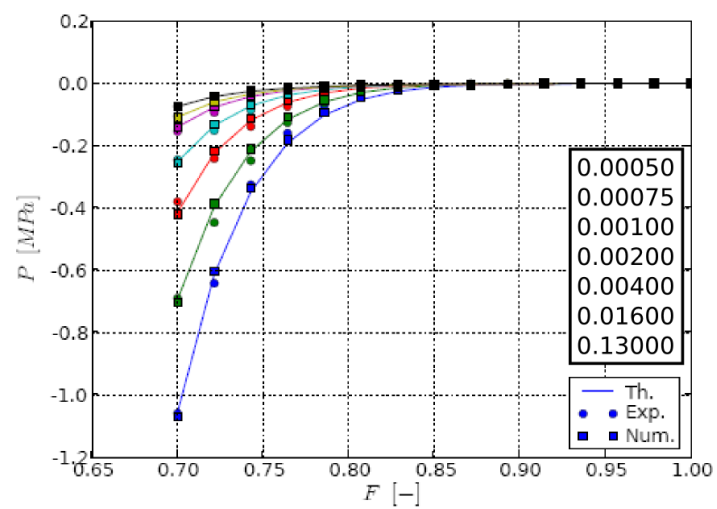

Figure 1: Nominal stress vs Gradient of deformation in uni-axial compression and at different strain-rates [1/s].

\section{Discussion}

The proposed dissipation potential is limited to isotropy but can be extended using the framework set forth by [Spencer, 1954]. It is required that the material responds asymptotically at high strainrates without any evidence of damage. We limited ourselves to the short-memory response of the material, the complete theoretical framework is available in the paper of [Pioletti, 2000]. Under particular cyclic strain condition and combined with large viscous contribution, long-time contribution must be taken into account as the description may deviate from realistic behaviour. When the material is not subjected to appreciable turn over a macroscopic phenomenological description of living tissues appears sufficient for joint stability analysis and eventually traumatic investigation during sport injuries. Mixture theory may be more appropriate to gain insight in biological response problems. The presented procedure enables one to conduct 'effortlessly' a viscoelastic description from experimentation to finite element implementation. The multi-modelling capability and the introduction of descriptive viscous material properties greatly simplifies the task of describing and contrasting different strainrate sensitive materials.

\section{References}

Pioletti et al, Eur J Mech A/S, 19: 749-759, 2000 Spencer, Theory of Invariants, New York, Academic Press, 1954. 